\title{
Le Programme de gestion thérapeutique des médicaments : 10 ans de collaboration entre les cinq centres hospitaliers universitaires du Québec
}

\author{
par France Varin, Martin Turgeon et Marie-Claude Michel
}

\section{INTRODUCTION}

T e Programme de gestion thérapeutique des médicaments (PGTM) est né d'une initiative des cinq centres hospitaliers universitaires (CHU) du Québec soit le Centre universitaire de santé McGill, le centre hospitalier de l'Université de Montréal, le Centre hospitalier universitaire de Québec, le Centre hospitalier universitaire mère-enfant Sainte-Justine et le Centre hospitalier universitaire de Sherbrooke.

Le PGTM a pour mandat de favoriser l'utilisation optimale des médicaments dans les $\mathrm{CHU}$ au moyen d'activités de soins, d'enseignement, de recherche et d'évaluation des technologies. C'est une entité entièrement autonome, qui appartient aux CHU et est indépendante du ministère de la Santé et des Services sociaux.

Depuis le début des années 2000, on assiste à une importante croissance du coût des médicaments dans les centres hospitaliers ${ }^{1,2}$.

L'ajout constant de nouvelles entités à l'arsenal thérapeutique exigeait que les comités de pharmacologie de chacun des $\mathrm{CHU}$ effectuent des analyses afin d'être en mesure de procéder à des choix éclairés. Ce travail d'évaluation en double nécessitait une utilisation importante de ressources, tant sur le plan médical que pharmaceutique et devenait d'autant plus problématique dans un contexte de pénurie de main d'œuvre.

Ces enjeux ont incité les cinq CHU du Québec à unir leurs efforts pour créer le PGTM. En mettant en commun une partie de leurs ressources et de leurs expertises, les $\mathrm{CHU}$ ont voulu se concerter pour assurer l'évaluation de nouveaux médicaments et favoriser un meilleur suivi de leur utilisation. Notre article vise à présenter ce programme ainsi que ses réalisations depuis les dix dernières années.

\section{DESCRIPTION DU PROGRAMME}

Le PGTM est un programme provincial regroupant des médecins et des pharmaciens de chacun des $\mathrm{CHU}$, dont le but est de favoriser l'utilisation optimale des médicaments dans leurs établissements. Pour le PGTM, la gestion thérapeutique se définit comme une approche systématique d'évaluation des médicaments permettant d'envisager leur ajout aux différentes listes locales et allant jusqu'à la mesure de leurs effets sur des groupes de patients. La gestion thérapeutique intègre des revues systématiques de la littérature médicale ainsi que des recherches évaluatives sur les pratiques entourant l'utilisation des médicaments, sur la mesure des retombées des interventions réalisées et également sur les coûts des soins de santé. La gestion thérapeutique vise également le développement d'outils d'aide à la décision, l'élaboration de méthodes d'intervention ainsi que la diffusion de l'information auprès des cliniciens en plus d'assurer une vigie des thérapies émergentes. Les principes directeurs du PGTM sont énumérés dans la boîte 1 .

Le PGTM a entrepris ses activités en 2004, à la suite d'une entente de partenariat intervenue entre les chefs de département de pharmacie et les directeurs des services professionnels des cinq CHU québécois. L'année 2004 a été consacrée au développement du Programme, à la formation des membres affectés au PGTM, à la mise en place de structures et à l'élaboration d'un guide méthodologique visant la standardisation des processus ${ }^{3}$. Pendant cette période, un processus uniformisé d'évaluation des médicaments a vu le jour. L'évaluation des médicaments au PGTM comporte deux volets : premièrement, des évaluations systématiques des données probantes qui mènent à la formulation de recommandations visant à orienter la réflexion des comités de 
Boîte 1. Principes directeurs du Programme de gestion thérapeutique des médicaments (PGTM)

Collaboration - réelle et équitable entre les $\mathrm{CHU}$ et entre les intervenants (p. ex. médecins, pharmaciens)

Validité scientifique - par l'utilisation de méthodes d'évaluation appropriées basée sur les données probantes

Transparence - au cours du processus par la prise en compte des contraintes légales

Pertinence - de la démarche due au développement d'outils qui répondent aux besoins des cliniciens et qui facilitent la prise de décision ainsi qu'à la présentation d'activités d'enseignement

Rayonnement - dans le monde scientifique afin que les intervenants du réseau en profitent et que le PGTM puisse bénéficier d'une reconnaissance par les pairs

Continuité - par le développement de méthodes, de bases de données et d'outils favorisant une évaluation continue de I'utilisation des médicaments ciblés

Éthique - par le respect des principes éthiques

pharmacologie et deuxièmement, des évaluations de l'utilisation des médicaments, notamment des analyses descriptives et des revues d'utilisation de médicaments.

\section{RESSOURCES ET FONCTIONNEMENT}

Le PGTM compte sept pharmaciens, représentant cinq équivalents temps complet, issus des cinq CHU. Les pharmaciens œuvrant au PGTM démontrent un intérêt soutenu pour la gestion thérapeutique, la recherche évaluative, l'évaluation de l'utilisation des médicaments et l'évaluation fondée sur les données probantes. Tous sont détenteurs d'une maîtrise en sciences et ils ont acquis des connaissances plus ciblées dans ces domaines à l'aide des programmes de formation spécifique. Ils sont nommés par le chef du département de pharmacie. Les pharmaciens participent à l'élaboration des documents et à la conduite des évaluations de médicaments. En une année, le PGTM réalise un nombre d'évaluations qui varie en fonction de leur ampleur. Il mène en moyenne deux revues d'utilisation ou analyses descriptives et présente annuellement trois à cinq rapports d'évaluation.

Des experts externes révisent les évaluations produites par les pharmaciens. Lorsque cela s'avère nécessaire, le PGTM a également recours à un biostatisticien ou à un épidémiologiste. Par la suite, un comité scientifique approuve les évaluations, et un comité exécutif les entérine.

Le comité scientifique se compose d'un médecin et du pharmacien coordonnateur du PGTM de chaque CHU (nommé par le chef de département lorsqu'il y a plus d'un pharmacien d'un même CHU, qui participe au Programme). Il a comme mandat de développer et de partager les expertises cliniques, scientifiques et méthodologiques. Il valide le contenu scientifique des documents et émet des recommandations. Il s'assure également de promouvoir et de maintenir à jour le guide méthodologique du PGTM. Le comité scientifique se réunit environ quatre fois par année.

Le comité exécutif, composé du directeur des services professionnels et du pharmacien chef de chaque CHU, entérine les recommandations sur l'utilisation des médicaments et s'assure d'une approche concertée des évaluations entre les cinq CHU. Ce comité établit ses échéanciers, les priorités pour les cinq CHU parmi les projets proposés et surveille les enjeux légaux, administratifs ou économiques. Finalement, il est responsable des publications ou des communications avec les instances internes ou externes. Les membres du comité exécutif se réunissent au besoin, soit en personne, soit par téléconférence. La fréquence des réunions peut varier selon l'urgence et le type des travaux en cours. Un processus d'approbation par courriel des différents documents est prévu.

En 2013, la création d'un poste d'adjoint s'est avérée nécessaire pour assurer la planification et la coordination des activités scientifiques et administratives du PGTM. Les ressources humaines et les activités du PGTM sont financées à parts égales par les cinq $\mathrm{CHU}$, et les fonds proviennent du budget opérationnel de chacun d'eux.

La figure 1 présente l'organigramme du PGTM.

\section{ÉVALUATION DU PROGRAMME}

Depuis sa création, le PGTM a effectué 36 évaluations de médicaments, deux revues de traitement et 27 analyses descriptives

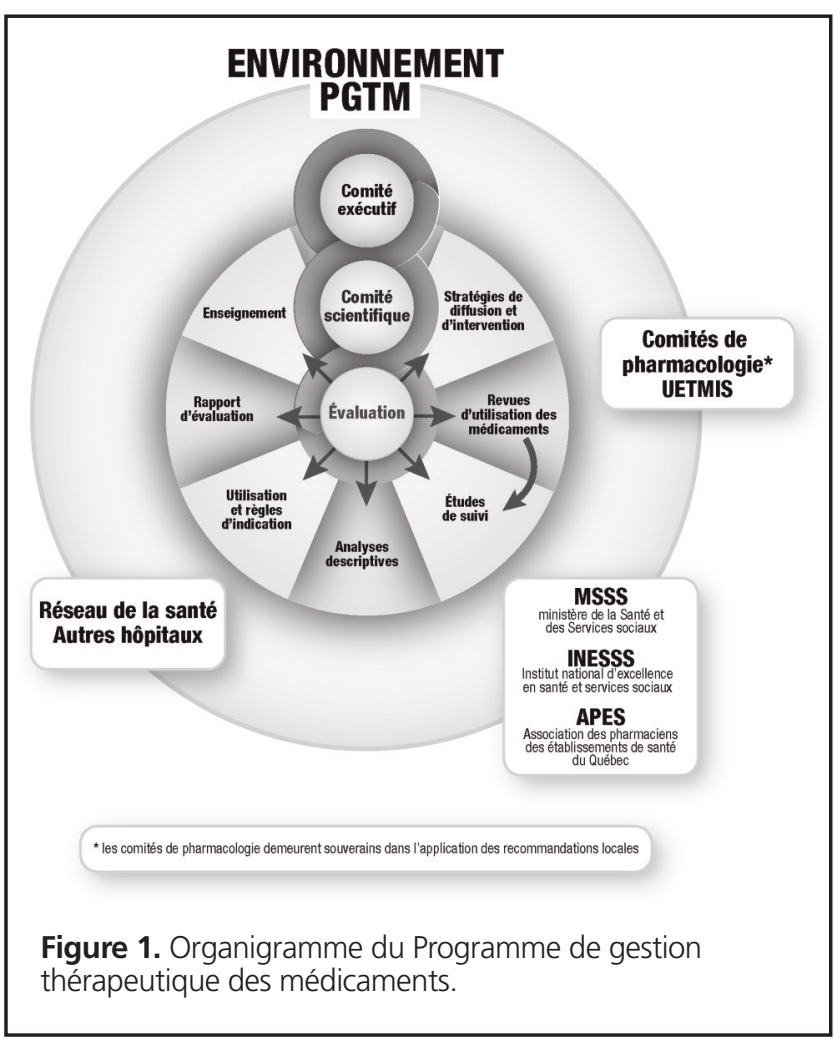


ou revues d'utilisation de médicaments ${ }^{4}$. Les recommandations sont accueillies favorablement et suivies intégralement à plus de $90 \%$ par les comités de pharmacologie.

L'expérience acquise au fil des ans par le PGTM a permis de mettre en lumière certaines difficultés liées à la diffusion de l'information et à la mise en application des recommandations émises à la suite des évaluations. Le partage des problèmes rencontrés et la mise en commun des solutions implantées dans chacun des $\mathrm{CHU}$ ont mené à l'élaboration d'un modèle d'intervention clinique (MIC). C'est ainsi que le Programme s'est donné comme objectif de développer un modèle de transfert des connaissances et de suivi des recommandations, nommé MIC. Celui-ci consiste en la mise en place d'outils efficaces et utiles aux cliniciens pour assurer l'usage optimal des médicaments ${ }^{4}$. En 2012, le PGTM a procédé à une revue systématique de la littérature scientifique sur les différentes méthodes d'interventions afin de déterminer celles jugées efficaces pour modifier le comportement des professionnels. Chaque projet effectué par le PGTM nécessite désormais le développement d'un MIC. Ce dernier est joint aux recommandations conformément aux critères issus de cette revue et de l'expérience vécue à travers les différentes réalisations et les divers engagements du PGTM au sein des CHU. Ce modèle contribue à l'évaluation de la qualité du transfert de connaissances ainsi que des retombées des recommandations.

L'engagement du pharmacien à développer des MIC et à les appliquer dans son milieu s'ajoute aux évaluations de médicaments. Chaque milieu priorise les projets pour lesquels il est important de s'investir, selon les problèmes rencontrés localement. Plusieurs MIC sont donc en cours simultanément et peuvent durer de quelques mois à quelques années.

Les membres du PGTM participent à titre de conférenciers à des activités de formation destinées principalement aux pharmaciens des hôpitaux du Québec, portant autant sur des sujets cliniques (ex. : antibioprophylaxie chirurgicale, revue du traitement des infections à SARM pour les patients hospitalisés) que sur des sujets à caractère plutôt méthodologique (ex. : comment conduire des analyses descriptives et des revues d'utilisation de médicaments). En tant qu'acteur contribuant au transfert des connaissances et au rayonnement des $\mathrm{CHU}$, le PGTM diffuse les résultats de ses études dans divers congrès locaux, provinciaux et internationaux, au moyen d'articles ${ }^{5-10}$, d'affiches et de conférences.

Vous trouverez au tableau 1 des exemples de réalisations du PGTM.

\section{RETOMBÉES SUR LA PRESTATION DES SOINS EN ÉTABLISSEMENT DE SANTÉ}

Les projets du PGTM entraînent des retombées importantes dans plusieurs secteurs des CHU. Leur portée peut être d'ordre clinique, économique, administratif ainsi qu'organisationnel et peut également toucher la sécurité du patient. Un seul projet peut ainsi avoir des répercussions positives dans plusieurs sphères.
Par exemple, afin d'assurer l'utilisation optimale de la dexmédétomidine à l'unité des soins intensifs, le PGTM a évalué, en 2010, les indications selon les données probantes et a émis une règle d'utilisation pour les $\mathrm{CHU}$. Cette règle s'avérait nécessaire pour ces établissements qui se trouvaient dans un contexte d'optimisation des dépenses, étant donné le coût dix fois plus élevé de la dexmédétomidine par rapport au traitement conventionnel. La conception d'une ordonnance préimprimée adaptée à chaque $\mathrm{CHU}$ ainsi qu'une revue d'utilisation des médicaments selon les critères préalablement établis après un an d'utilisation ont été mis de l'avant. L'approche proactive préconisée par le PGTM pour l'usage de la dexmédétomidine a permis non seulement l'encadrement clinique de son utilisation par les cliniciens et la rétroaction nécessaire à l'apport de tout correctif éventuel, mais également un meilleur contrôle des coûts.

Par ailleurs, certains dossiers traités par le PGTM touchent des questions ayant des conséquences administratives importantes pour les CHU. Par exemple, une analyse descriptive de la gestion des médicaments utilisés dans le cadre du Programme d'accès spécial de Santé Canada a mis en lumière certaines améliorations possibles touchant des aspects de la gestion des demandes et du suivi nécessaire pour l'usage de ces médicaments. L'analyse descriptive portant sur les ruptures d'approvisionnement en médicaments survenues en 2012 a permis de quantifier leurs conséquences sur les ressources humaines et financières, et de soulever des aspects cliniques et organisationnels majeurs qui pourront guider les décisions futures ainsi que les mesures à prendre face aux ruptures d'approvisionnement de médicaments.

Quant à la sécurité des patients, certaines analyses descriptives ont pu déterminer des lacunes dans l'utilisation sécuritaire des médicaments. Une analyse descriptive de l'utilisation des opioïdes a permis notamment de découvrir certaines caractéristiques non conformes dans les écarts de doses prescrites et a facilité la mise en application de mesures correctrices améliorant la sécurité de la prescription de ces médicaments. En vue d'assurer le suivi des modifications apportées, les CHU ont répété cette analyse à plusieurs reprises. Une revue de l'utilisation des abréviations lors de la rédaction des ordonnances a aussi aidé les CHU à déterminer certaines pratiques susceptibles d'amélioration, relatives à la qualité et à la sécurité des soins. L'élaboration d'un plan d'action concerté entre les cinq CHU (campagne de sensibilisation, audit et rétroaction) a permis de cibler et de prioriser les interventions propres à maximiser les résultats.

En plus de positionner dans la thérapie les protocoles Folfox, Folfiri et Xelox pour le traitement du cancer colorectal métastatique, cette évaluation a favorisé une meilleure gestion des coûts et de l'organisation des services offerts aux cliniques d'oncologie dans les CHU. Cette revue a également permis d'établir une trajectoire de soins sécuritaire et optimale pour les patients.

Certains dossiers traités par le PGTM ont aussi contribué à établir un portrait de quelques pratiques ayant cours dans les $\mathrm{CHU}$, pour permettre l'évaluation du degré de conformité des 
This single copy is for your personal, non-commercial use only.

For permission to reprint multiple copies or to order presentation-ready copies for distribution, contact CJHP at cjhpedit@cshp.ca

\section{Tableau 1. Exemples de travaux effectués par le Programme de gestion thérapeutique des médicaments depuis sa création}

Titre

Évaluation de Revue d'utilisation médicaments

de médicaments

ou analyse

descriptive

\begin{tabular}{|c|c|c|c|c|}
\hline \multirow{2}{*}{\multicolumn{2}{|c|}{$\begin{array}{l}\text { Abréviations, } 2013 \\
\text { Antibioprophylaxie chirurgicale }\end{array}$}} & $\sqrt{ }$ & & Affiches (APES, RQRUM) \\
\hline & & $\sqrt{ }$ & & $\begin{array}{l}\text { Affiche (ICAAC 2014), formation } \\
\text { Internet (2008) }\end{array}$ \\
\hline $\begin{array}{l}\text { Antibioprophylaxie de l'endocardite } \\
\text { bactérienne, } 2012\end{array}$ & & & $\sqrt{ }$ & $\begin{array}{l}\text { Guides cliniques en antibiothérapie, } \\
\text { Série II: antibioprophylaxie de } \\
\text { l'endocardite bactérienne }{ }^{11} \text { (INESSS) }\end{array}$ \\
\hline $\begin{array}{l}\text { Azacitidine pour le traitement du } \\
\text { syndrome myélodysplasique et de } \\
\text { la leucémie myéloïde aiguë, } 2011\end{array}$ & $\sqrt{ }$ & $\sqrt{ }$ & & \\
\hline $\begin{array}{l}\text { Dabigatran en fibrillation auriculaire, } \\
2011 \text { et pour la chirurgie } \\
\text { orthopédique, } 2009\end{array}$ & $\sqrt{ }$ & & & Conférence (APES) \\
\hline $\begin{array}{l}\text { Dexmédétomidine (Précédex) pour } \\
\text { l'adulte et en pédiatrie, 2010; pour } \\
\text { la sédation consciente, } 2011\end{array}$ & $\sqrt{ }$ & $\sqrt{ }$ & & $\begin{array}{l}\text { Affiche (APES), article }{ }^{5}, \\
\text { conférence (APES) }\end{array}$ \\
\hline $\begin{array}{l}\text { Folfiri, Folfox et Xelox pour le } \\
\text { cancer du côlon métastatique, } 2011\end{array}$ & & & $\sqrt{ }$ & \\
\hline Méropénem 2011 & & $\sqrt{ }$ & & Affiche (ICAAC) \\
\hline $\begin{array}{l}\text { Les narcotiques phase I-II-III, } \\
2006 \text { à } 2012\end{array}$ & & $\sqrt{ }$ & & Affiches (ACMTS, APES), article ${ }^{9}$ \\
\hline $\begin{array}{l}\text { PAS : Médicaments obtenus par le } \\
\text { Programme d'accès spécial de } \\
\text { Santé Canada, } 2010\end{array}$ & & $\sqrt{ }$ & & Affiches (APES, PPC/SCPH) \\
\hline
\end{tabular}

Pertuzumab pour le traitement du

Revue de traitements

Rayonnement externe aux CHU

cancer du sein métastatique

HER-2 positif, 2014

Le Programme de gestion

thérapeutique des médicaments

Revue des antibiotiques utilisés pour

le traitement d'une infection à

S. aureus résistant à la méthicilline

chez le patient adulte hospitalisé,

2012

\begin{tabular}{|c|c|c|c|}
\hline Rituximab, 2006 à 2014 & $\sqrt{ }$ & $\sqrt{ }$ & $\begin{array}{l}\text { Affiche (ASH 2010), conférence } \\
\text { (CLOPPE 2010) }\end{array}$ \\
\hline $\begin{array}{l}\text { Rupture d'approvisionnement en } \\
2012 \text { dans les CHU du Québec }\end{array}$ & & $\sqrt{ }$ & $\begin{array}{l}\text { Affiche (PPC/SCPH), conférence } \\
\text { (PPC/SCPH) }\end{array}$ \\
\hline $\begin{array}{l}\text { Trastuzumab pour le traitement du } \\
\text { cancer du sein adjuvant, 2007, } 2009\end{array}$ & $\sqrt{ }$ & $\sqrt{ }$ & Affiche (ASCO) \\
\hline $\begin{array}{l}\text { L'utilisation optimale des } \\
\text { médicaments par la revue } \\
\text { d'utilisation médicamenteuse et le } \\
\text { transfert des connaissances, } 2007\end{array}$ & & & $\begin{array}{l}\text { Ateliers de formation pour les } \\
\text { pharmaciens du Québec }\end{array}$ \\
\hline \multicolumn{4}{|c|}{$\begin{array}{l}\text { ACMTS = Agence canadienne des médicaments et des technologies de la santé, APES = Association des pharmaciens des } \\
\text { établissements de santé, ASCO = American Society of Clinical Oncology, ASH = American Society of Hematology, } \\
\mathrm{CHU}=\text { Centre hospitalier universitaire, CLOPPE = Club de lecture des pharmaciens en oncologie du Québec, FSI = Forum Santé } \\
\text { International, HTAi = Health Technology Assessment International, ICAAC = Interscience Conference on Antimicrobial and } \\
\text { Chemotherapy, INESSS = Institut national d'excellence en santé et services sociaux, PPC/SCPH = Conférence annuelle sur } \\
\text { la pratique professionnelle/Société canadienne des pharmaciens d'hôpitaux, RQRUM = Réseau québécois de recherche sur } \\
\text { I'usage des médicaments. }\end{array}$} \\
\hline
\end{tabular}

pratiques organisationnelles requises par Agrément Canada. Ainsi, les CHU ont pu déterminer les corrections à apporter pour répondre à ces pratiques organisationnelles requises. Les analyses descriptives portant sur les prescriptions d'opiö̈des et sur l'utilisation des abréviations dites dangereuses en sont des exemples. Par ailleurs, la réalisation d'une revue de la littérature médicale sur l'héparine et des héparines à bas poids moléculaire a abouti à des recommandations sur l'usage sécuritaire de ces molécules dans nos CHU.

Il importe de souligner que les résultats des analyses descriptives et des revues d'utilisation des médicaments permettant une comparaison des données locales avec celles d'autres $\mathrm{CHU}$ 
confrontés à des missions ainsi qu'à des enjeux cliniques et économiques semblables suscitent un vif intérêt chez les professionnels de la santé .

La diffusion de l'information étant au cour des préoccupations du PGTM, les documents du PGTM ainsi que le guide méthodologique peuvent être consultés sur son site web à l'adresse suivante www.pgtm.qc.ca. Les autres centres hospitaliers de la province, qui ne possèdent pas toutes les ressources du PGTM, peuvent ainsi utiliser ces documents pour aider leur comité de pharmacologie à la prise de décision et les outiller pour la conduite d'une revue d'utilisation des médicaments.

Finalement, le PGTM collabore avec des d'organismes provinciaux reconnus, tels l'Institut national d'excellence en santé et en services sociaux, l'Association des pharmaciens en établissement de santé et le Comité de l'évolution de la pratique en oncologie. Le PGTM a collaboré avec ces organismes à la création de guides de pratique ${ }^{11}$ favorisant une optimisation de la thérapie et à l'élaboration d'activités de formation continue ${ }^{12,13}$. Le PGTM participe également aux travaux de certaines unités d'évaluation des technologies et des modes d'intervention en santé présentes dans les $\mathrm{CHU}^{14}$.

\section{CONCLUSION}

Au fil des ans, plusieurs facteurs ont contribué au succès du Programme. Une méthodologie commune et rigoureuse, une communication continuelle et une réelle collaboration entre les CHU ont permis de relever les différents défis. La diffusion des travaux à tous les professionnels de la santé a favorisé une reconnaissance plus large du Programme. À notre connaissance, un tel programme issu d'une réelle collaboration multidisciplinaire entre plusieurs hôpitaux universitaires est unique.

Plusieurs enjeux demeurent toutefois présents. Les nombreux projets en cours nécessitent un engagement constant pour parachever chacun d'eux. L'émergence continuelle de nouvelles molécules demeure un défi de taille pour l'émission de recommandations en temps opportun et selon les besoins des cliniciens et des comités de pharmacologie de chaque CHU.

Par ses différents projets, le PGTM fournit aux cliniciens une mise à jour des informations basées sur une analyse rigoureuse des données probantes afin d'optimiser l'utilisation des médicaments dans les CHU du Québec. Il propose des modèles d'interventions cliniques et offre des outils d'aide à la décision pour faciliter le transfert de la théorie vers un changement de pratique dans les milieux. En raison des ressources professionnelles limitées et des coûts des médicaments sans cesse grandissants, les CHU du Québec ont uni leurs expertises et leurs ressources pour optimiser la pharmacothérapie des patients. Après dix années d'existence, le Programme connaît un réel succès, et les sujets pour alimenter ses travaux futurs sont toujours au rendez-vous.
Références

1. Allard G. Coût des médicaments. I-Média (Bulletin d'information de l'Association québécoise d'établissements de santé et de services sociaux). 22 novembre 2012. Publié au: www.aqesss.qc.ca/1783/editions_anterieures. aqesss?id=445. Consulté le 24 novembre 2014.

2. Allard G. Zoom sur le coût des médicaments. I-Média (Bulletin d'information de l'Association québécoise d'établissements de santé et de services sociaux). 19 décembre 2013. Publié au : www.aqesss.qc.ca/1783/editions_anterieures. aqesss?id=500. Consulté le 24 novembre 2014.

3. Bussières JF. Un premier programme conjoint de gestion thérapeutique des médicaments entre les CHU. Artère. 2004 juillet-août;22:4 -5.

4. Le Programme de gestion thérapeutique des médicaments. Publié au : http:// pgtm.qc.ca/methodes-dintervention-clinique-2/. Consulté le16 novembre 2014.

5. Turgeon M, Pelletier E, Bérard G, Guévremont C, Marcotte N, Michel $\mathrm{MC}$, et al. La conduite de revues d'utilisation de médicaments : un exercice qui gagne à être répété [lettre]. Pharmactuel. 2014;47(3):152-3.

6. Michel MC. Infection nosocomiale à Staphylococcus aureus résistant à la méthicilline chez un patient adulte hospitalisé : quel antibiotique choisir ? Pharmactuel. 2013;46(1):23-33.

7. Michel MC. Comité de pharmacologie ... comment gérer les antibiotiques? Pharmactuel. 2009;42 Suppl 1:33-40.

8. Cossette B, Turgeon M, Letarte N, Dupont C. Doit-on inclure des données non publiées (abrégés de congrès) dans les évaluations des comités de pharmacologie? Pharmactuel. 2007;40(5):281-6.

9. Dupont C. Description des ordonnances actives de narcotiques dans les centres hospitaliers universitaires du Québec - un projet du Programme de gestion thérapeutique des médicaments. Pharmactuel. 2007;40 Suppl 2:19-22.

10. Cossette B, Letarte N, Dehault C. Optimiser la recherche de littérature pour obtenir le plus de références pertinentes sur un sujet de pharmacothérapie. Pharmactuel. 2005;38(3):140-6.

11. Guides cliniques en antibiothérapie, Série II: antibioprophylaxie de l'endocardite bactérienne. Québec (QC) : Institut national d'excellence en santé et services sociaux; 2012. Publié au : www.inesss.qc.ca/fileadmin/doc/ INESSS/Outils/Guides_antibio_II/endocardite_2012_web_FR.pdf. Consulté le 26 novembre 2014.

12. Activités de formation continue passées, 2008. Montréal (QC) : Association des pharmaciens des établissements de santé du Québec. Publié au : www. apesquebec.org/app/wa/mediaEntry?mediaEntryId=2764. Consulté le 26 novembre 2014.

13. Activités de formation continue passées, 2011. Montréal (QC) : Association des pharmaciens des établissements de santé du Québec; 2010. Publié au : www.apesquebec.org/app/wa/mediaEntry?mediaEntryId=3439. Consulté le 26 novembre 2014.

14. Gouvernance. Québec (QC) : Centre hospitalier universitaire de Québec, Unité d'évaluation des technologies et des modes d'intervention en santé. Publié au : www.chuq.qc.ca/fr/evaluation/uetmis/gouvernance/. Consulté le 26 novembre 2014.

France Varin, B. Pharm., M. Sc., travaille au Département de pharmacie, Centre hospitalier de l'Université de Montréal, Montréal, Québec.

Martin Turgeon, B. Pharm., M. Sc., travaille au Département de pharmacie, Centre hospitalier universitaire de Sherbrooke - Hôtel-Dieu, Sherbrooke, Québec.

Marie-Claude Michel, B. Pharm., M. Sc., travaille au Département de pharmacie, Centre hospitalier universitaire de Québec, Sainte-Foy, Québec.

Intérêts concurrents : Aucun déclaré.

\section{Adresse de correspondance :}

France Varin

Département de pharmacie

Centre hospitalier de I'Université de Montréal

190, boulevard René Lévesque Est, bureau 306

Montréal QC H2X $1 \mathrm{~N} 5$

Courriel : france.varin.chum@ssss.gouv.qc.ca

Remerciements : Nous remercions Mme Nathalie Marcotte, Mme Chantal Guévremont et M Ghislain Bérard pour la révision du document. 